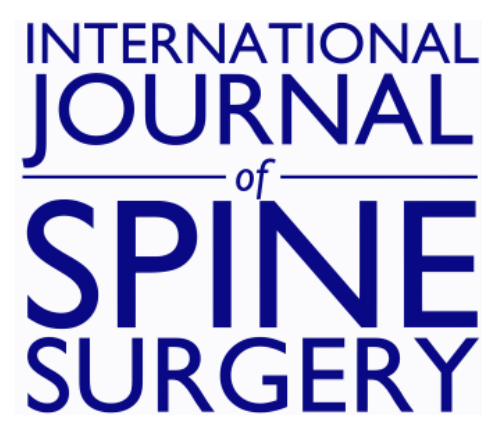

\title{
Osteopenia and Total Disc Prosthesis Subsidence: Inclusion/Exclusion Criteria for Total Disc Replacement
}

Casey K. Lee

Int J Spine Surg 2007, 1 (2) 82-84

doi: https://doi.org/10.1016/SASJ-2007-0104-RR

http://ijssurgery.com/content/1/2/82

This information is current as of April 26, 2023.

Email Alerts Receive free email-alerts when new articles cite this article. Sign up at:

http://ijssurgery.com/alerts

The International Journal of Spine Surgery

2397 Waterbury Circle, Suite 1,

Aurora, IL 60504, Phone: +1-630-375-1432

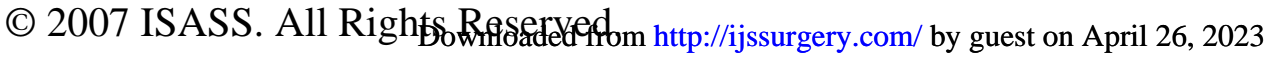




\title{
Osteopenia and Total Disc Prosthesis Subsidence: Inclusion/Exclusion Criteria for Total Disc Replacement
}

\author{
Casey K. Lee, MD
}

\begin{abstract}
Background

Prosthetic subsidence is an adverse event of disc arthroplasty with a total disc prosthesis. Factors affecting prosthetic subsidence are (1) the size of the prosthetic endplate (footprint), (2) amount of load applied to the spine, and (3) compressive strength of the vertebral bone. The size of the prosthetic endplate has been addressed adequately in the development of the current generation of total disc prostheses. However, little information is available on the relation between osteopenia (low bone mineral density [BMD]) and prosthetic subsidence. We evaluated the relation between osteopenia and compressive strength of vertebral bone and propose an inclusion/exclusion guideline for disc arthroplasty in the presence of osteopenia.
\end{abstract}

\begin{abstract}
Methods
This study was based on previously published data by C.K. Lee on bone mineral density, contact surface area, and applied load that emphasized the critical contact surface area required to prevent subsidence. The study included 35 cadaveric vertebral bones (representing ages 38-68) that were evaluated via quantitative computed tomography for BMD and subjected to nondestructive and destructive tests for compressive strength of the vertebral bones. The relationship of osteopenia to prosthetic subsidence was evaluated from the data.

Results

Patients with average BMD $\left(0.137 \mathrm{~g} / \mathrm{cm}^{3}\right)$ require a minimum contact surface area of $6.5 \mathrm{~cm}^{2}$ for the physiologic load of $2500 \mathrm{~N}$. Patients with a BMD of up to 1 SD below the average will require $9.1 \mathrm{~cm}^{2}$ of contact area for the same load. Patients with a BMD of up to 2 SD below the average will require 13 $\mathrm{cm}^{2}$ of contact area.

Discussion

The average endplate surface area of small disc prostheses is about $6.5-8 \mathrm{~cm}^{2}$, which is enough to tolerate normal physiologic load for patients with normal BMD. Patients with BMD of 2 SD below the average will require a disc prosthesis with an endplate surface area greater than $12.75 \mathrm{~cm}^{2}$ to tolerate normal physiologic load. The largest size of prosthetic endplate of the currently available disc prostheses is about $11 \mathrm{~cm}^{2}$; therefore, patients with BMD below 2 SD will have a high risk of subsidence, and these patients should be excluded for disc arthroplasty. Patients with BMD between -1.5 SD and -2.0 SD may be included for disc arthroplasty only when they can receive the large size of prosthesis with greater than $10.5 \mathrm{~cm}^{2}$ prosthetic endplates.

Conclusions

All patients should be evaluated preoperatively with BMD measurement. Patients with a BMD of up to 1.5 SD below the average can be included for total disc arthroplasty. Patients with BMD of 2 SD below the average should be excluded for total disc arthroplasty, and patients with BMD of 1.5-2 SD below the average should be carefully evaluated for the appropriate size of the prosthesis prior to the surgery.
\end{abstract}

Key Words disc arthroplasty, subsidence, osteopenia. SAS Journal. Spring 2007;1:82-84. DOI: SASJ-2007-0104-RR

\section{INTRODUCTION}

Subsidence of total disc prosthesis is an adverse event of disc arthroplasty that may range from subtle subsidence to a disastrous vertebral bone fracture underneath the disc prosthesis. The 3 most important factors affecting prosthetic subsidence are (1) the size of the prosthetic endplate (footprint), (2) amount of load to the spine, and (3) compressive strength of the vertebral bone. The relationship between these 3 factors has been studied and the importance of the critical contact surface area (footprint) has been well recognized in the past. ${ }^{1}$ However, little 
information is available regarding the severity of osteopenia and prosthetic subsidence. The purpose of this study is to reevaluate the relation between osteopenia and compressive strength of vertebral bone, and to propose an inclusion/exclusion guideline for osteopenia in disc arthroplasty.

\section{MATERIALS AND METHODS}

This study was based on data I previously published on bone mineral density (BMD), contact surface area, and applied load that emphasized the critical contact surface area to prevent subsidence. ${ }^{1}$ The study was based on 35 cadaveric thoracic vertebral bones (from patients aged 38-68) that were evaluated via quantitative computed tomography for BMD and subjected to nondestructive and destructive tests for compressive strength of the vertebral bones. The vertebral endplates were carefully decorticated to ensure total contact surface with the load application block with known surface areas. My previous study was conducted for the load range of 600-2000 N, contact surface areas of $1.1 \mathrm{~cm}^{2}, 2.4 \mathrm{~cm}^{2}$, and $4.0 \mathrm{~cm}^{2}$ (which correspond to $15 \%, 33 \%$, and $55 \%$ of the vertebral endplate surface area), and BMD of $\pm 1.5 \mathrm{SD}$. The current evaluation is an extrapolation of the previous study for the ranges of the contact surface areas up to $13 \mathrm{~cm}^{2}$, BMD of $-2 \mathrm{SD}$, and load up to $3000 \mathrm{~N}$ for the lumbosacral spine. The relation of osteopenia and prosthetic subsidence was evaluated for the specific range of $8-11 \mathrm{~cm}^{2}$ of the contact surface area, the range of prosthetic endplate size for current disc prostheses.

\section{RESULTS}

The results of the current evaluation are shown in Figure 1. The current generation of disc prostheses has a prosthetic endplate size of $8-11 \mathrm{~cm}^{2}$. The ranges that I was interested in (indicated by bold rectangular lines in Figure 1) are the contact surface area between $8 \mathrm{~cm}^{2}$ and $11 \mathrm{~cm}^{2}$, the physiologic load of 2000$3000 \mathrm{~N}$, and BMD of normal to 3 SD below normal. Patients with $\mathrm{BMD}$ between the average and $-1.5 \mathrm{SD}$ can receive any size of disc prosthesis with the prosthetic endplate sizes ranging between $8 \mathrm{~cm}^{2}$ and $11 \mathrm{~cm}^{2}$ and will be able to tolerate $2500 \mathrm{~N}$. Patients with BMD below -2.0 SD (indicated by D1, D2, and D3 in Figure 1) will not tolerate the physiologic load even with the largest endplate size of currently available disc prostheses. Patients with BMD between -1.5 SD and -2.0 SD can tolerate a load of $2500 \mathrm{~N}$ only when they receive a disc prosthesis with the largest $\left(11 \mathrm{~cm}^{2}\right)$ endplate size (indicated by $\mathrm{C} 3$ in Figure 1). However, patients with BMD between -1.5 SD and -2.0 SD will not tolerate physiologic load if they receive an endplate size smaller than $10.5 \mathrm{~cm}^{2}$, because of a small vertebral body (indicated by $\mathrm{C} 1$ and $\mathrm{C} 2$ in Figure 1).

\section{DISCUSSION}

The incidence of subsidence of disc prosthesis was high (35\%) given the small endplate size of the Charite disc prosthesis I and II. The incident rate has decreased significantly with the larger endplate size of the Charité III (originally manufactured by Waldemar Link GmbH \& Co, Hamburg, Germany; now by DePuy Spine, Raynham, Mass). ${ }^{2}$ The importance of prosthetic

\section{Figure 1}

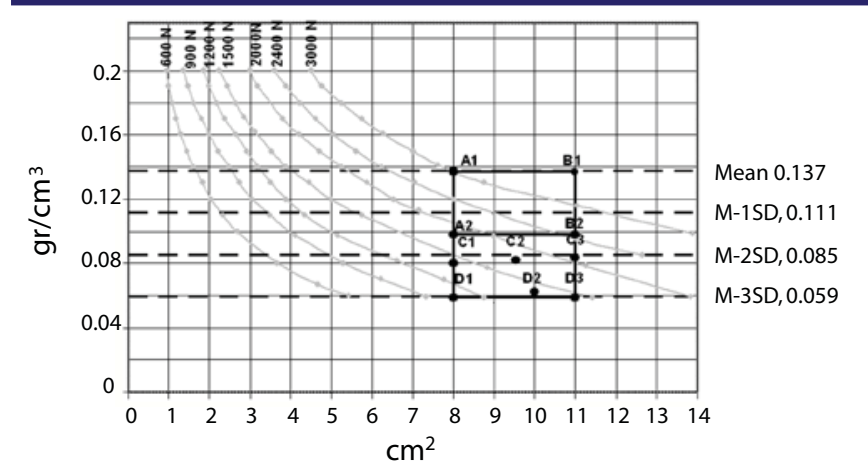

Note. The area within the rectangle represents the clinical area of interest in contact surface area of the range $8-11 \mathrm{~cm}^{2}$ (currently available prosthetic endplate sizes), osteopenia, and physiologic load on the lumbar spine (2000$3000 \mathrm{~N}$ ). All patients with bone mineral density (BMD) no more than 1.5 SD below average will be able to tolerate physiologic load for any size of prosthetic endplates between $8 \mathrm{~cm}^{2}$ and $11 \mathrm{~cm}^{2}$. No patient with BMD more than 2 SD below average will tolerate physiologic load even with the largest size of prosthetic endplate. Patients with BMD between $-1.5 \mathrm{SD}$ and $-2 \mathrm{SD}$ will tolerate physiologic load only when they receive prosthetic endplates greater than $10 \mathrm{~cm}^{2}$ in area. Patients in this range of BMD who receive endplates less than $10 \mathrm{~cm}^{2}$ in size will most likely develop subsidence (risk over $80 \%$ ).

Relation between BMD, applied load, and contact surface area of endplate.

endplate size has been well recognized. ${ }^{2}$ Endplate size among the current generation of total disc prostheses ranges from $8 \mathrm{~cm}^{2}$ to $11 \mathrm{~cm}^{2}$ for the small, medium, and large sizes. The incident rate for the current generation of total disc prostheses is not well documented, but it is estimated to be $3 \%$ to $5 \%$ for $3 \mathrm{~mm}$ or more subsidence. ${ }^{3}$ Considering the normal disc height is about $12 \mathrm{~mm}$, subsidence of $3 \mathrm{~mm}$ represents a loss of $25 \%$ of the disc height. Subsidence of this magnitude may significantly alter the biomechanics of the segment. Sporadic reports have been made of disastrous subsidence, including vertebral bone fractures, after total disc arthroplasty.

At present, no clear guidelines exist for inclusion/exclusion criteria for osteopenia. Although osteoporosis has been listed as an exclusion criterion in most clinical studies for disc arthroplasty, there is no clear recommendation of preoperative workups for BMD measurement.

This biomechanical study was conducted with decortication of the thin vertebral endplate to ensure the best contact surface area with the loading block. The thin cortical endplate contributes only $10 \%-15 \%$ of the compressive strength, ${ }^{4,5}$ and most of the compressive strength of the vertebral body is supported by the cancellous bone structures. Milling of the cortical endplates reduces compressive strength by about $10 \%$ but produces a greater amount of contact surface area with the prosthetic endplate. The surface of the vertebral endplate is very irregular and has a variable degree of concavity. ${ }^{6}$ If a flat-surfaced prosthetic endplate is fitted onto the unmilled, irregularly-shaped concave curvature of the vertebral endplate, the contact surface area will be very small at the periphery of the prosthetic endplate. Even with a large prosthetic endplate, the contact surface area with the concave and irregular surface of the vertebral bony endplate will be very small. Proper design 
of the prosthetic endplate or contouring of the vertebral bony endplate will increase the contact surface area between bone and prosthesis.

The average size of the vertebral endplate of the lumbar spine is about $14 \mathrm{~cm}^{2} .{ }^{7}$ Approximately $85 \%-90 \%$ of this area is covered by very thin cortical bone, and only the peripheral $2-5 \mathrm{~mm}$ is made of cortical bone on the exterior wall of the vertebral body. Almost all of the current disc prostheses are designed so that the prosthetic endplates do not come in contact with this peripheral cortical shell. To make the prosthetic endplate come in contact with the peripheral cortical shell, the prosthetic endplate has to be greater than $14 \mathrm{~cm}^{2}$ in area. This size may be impractical, requiring removal of entire annulus.

It is evident from our study that patients with osteopenia with BMD between normal and -1.5 SD will tolerate physiologic load even with a small prosthetic endplate. Patients with BMD below -2 SD will not tolerate the current generation of disc prostheses for physiologic load even with the largest size and should be excluded for disc arthroplasty. Patients with BMD between $-1.5 \mathrm{SD}$ and $-2.0 \mathrm{SD}$ will not tolerate small and medium-sized disc prostheses. These patients should be carefully evaluated preoperatively for the size of the vertebral endplates. Only patients with large vertebral bones that can receive the large disc prosthesis may be included for disc arthroplasty.

All patients should be evaluated preoperatively with BMD measurement. Patients with BMD of 1.5 SD below the average can be safely included for total disc arthroplasty. Patients with BMD of 1.5-2 SD below the average should be carefully evaluated for the appropriate size of the prosthesis the surgery, and patients with BMD of $2 \mathrm{SD}$ or more below the average should be excluded for total disc arthroplasty.

\section{C.K. Lee, MD}

From Spine Care and Rehabilitation, Roseland, New Jersey.

The author is a board member and founder of Nexgen Spine Inc, Whippany, New Jersey.

Address correspondence and reprint requests to Casey K. Lee, MD, Spine Care and Rehabilitation, 556 Eagle Rock Ave, Suite 5, Roseland, NJ, 07068 (email: caseykleemd@aol.com).

This submission was received June 12, 2007, and accepted for publication June 14, 2007.

Institutional review board approval was not required for this study.

\section{REFERENCES}

1. Closkey RF, Parsons JR, Lee CK, Blackshin MF, Zimmerman MC. Mechanics of interbody spinal fusion: analysis of critical bone graft area. Spine. 1993;18:1011-1015.

2. Blumenthal S, Geisler F, Guyer R, Ohnmeiss D. Similarities and differences in clinical and radiographic outcomes of two US IDE trials for lumbar disc replacement. Paper presented at the 10th Annual Meeting of the
Spine Arthroplasty Society; May 1-4, 2007; Berlin, Germany.

3. Griffith SL, Shelakov AP, Buttner-Janz, Le Maire JP, Zeegers WS. A multicenter retrospective study of the clinical results of the Link SB Charité intervertebral disc prosthesis: The initial European experience. Spine. 1994;19:1842-1849.

4. McBroom RJ, Hayes WC, Edwards WT, Goldberg RP, White AA III. Prediction of vertebral body compressive fracture using quantitative computer tomography. J Bone Joint Surg Am. 1985;67:1206-1214.

5. Hollowell JP, Vollmer DG, Wilson CR, Pintar FA, Yoganandan N. Biomechanical analysis of thoracolumbar interbody constructs. How important is the endplate? Spine. 1996;21:1032-1036.

6. Langrana NA, Kale SP, Edwards T, Lee CK, Kopacz K. Measurement of endplate curvatures for different species and study of curvature effect on the stress distribution in human lumbar motion segment. Spine J. 2006;6:267278.

7. Farfan HF, Cossette JW, Robertson GH, Wells RV, Kraus H. The effects of torsion on lumbar intervertebral joints: the role of torsion in the production of disc degeneration. J Bone Joint Surg Am. 1970;52A:468-497. 